\title{
On Rigid and Infinitesimal Rigid Displacements in Three-Dimensional Elasticity
}

\author{
Philippe G. CIARLET*† \\ Department of Mathematics \\ City University of Hong Kong \\ 83 Tat Chee Avenue \\ Kowloon, Hong Kong
}

\author{
Cristinel MARDARE $\ddagger$ \\ Laboratoire Jacques-Louis Lions \\ Université Pierre et Marie Curie \\ 4 Place Jussieu \\ 75005 Paris, France
}

\begin{abstract}
Let $\Omega$ be an open connected subset of $\mathbb{R}^{3}$ and let $\Theta$ be an immersion from $\Omega$ into $\mathbb{R}^{3}$. It is first established that the set formed by all rigid displacements, i.e., that preserve the metric, of the open set $\boldsymbol{\Theta}(\Omega)$ is a submanifold of dimension 6 and of class $\mathcal{C}^{\infty}$ of the space $\boldsymbol{H}^{1}(\Omega)$. It is then shown that the vector space formed by all the infinitesimal rigid displacements of the same set $\boldsymbol{\Theta}(\Omega)$ is nothing but the tangent space at the origin to this submanifold. In this fashion, the familiar "infinitesimal rigid displacement lemma" of three-dimensional linearized elasticity is put in its proper perspective.
\end{abstract}

MSC: 73C02, 73G99

Keywords: Submanifold, rigidity theorem, infinitesimal rigid displacement lemma, three-dimensional linearized elasticity

\footnotetext{
${ }^{*}$ Corresponding author

${ }^{\dagger}$ E-mail address: mapgc@cityu.edu.hk

${ }^{\ddagger}$ E-mail address: mardare@ann. jussieu.fr
} 


\section{Introduction}

Further details about the various notions and notations used here are provided in the next sections.

The following infinitesimal rigid displacement lemma in curvilinear coordinates plays a crucial rôle in linearized three-dimensional elasticity: Let $\Omega$ be an open connected subset of $\mathbb{R}^{3}$, let $\Theta$ be a smooth enough immersion from $\Omega$ into a three-dimensional Euclidean space $\mathbb{E}^{3}$, and let $\widetilde{\boldsymbol{v}} \in \boldsymbol{H}^{1}(\Omega)$ be a vector field that satisfies

$$
e_{i \| j}(\widetilde{\boldsymbol{v}})=0 \text { a.e. in } \Omega,
$$

where

$$
e_{i \| j}(\widetilde{\boldsymbol{v}})=\frac{1}{2}\left(\partial_{i} \widetilde{\boldsymbol{v}} \cdot \boldsymbol{g}_{j}+\partial_{j} \widetilde{\boldsymbol{v}} \cdot \boldsymbol{g}_{i}\right) \quad \text { and } \quad \boldsymbol{g}_{i}=\partial_{i} \boldsymbol{\Theta} .
$$

Then there exist vectors $\boldsymbol{c} \in \mathbb{E}^{3}$ and $\boldsymbol{d} \in \mathbb{E}^{3}$ such that

$$
\widetilde{\boldsymbol{v}}(x)=\boldsymbol{c}+\boldsymbol{d} \wedge \boldsymbol{\Theta}(x) \text { for almost all } x \in \Omega .
$$

For a proof, see [3, Theorem 1.7-3].

In elasticity theory in curvilinear coordinates, the set $\Theta(\Omega) \subset \mathbb{E}^{3}$ is viewed as the reference configuration of a three-dimensional elastic body and the field $\widetilde{\boldsymbol{v}}$ is viewed as a displacement field of the set $\boldsymbol{\Theta}(\Omega)$.

The functions $e_{i \| j}(\widetilde{\boldsymbol{v}})$ are the covariant components of the linearized change of metric tensor associated with the displacement field $\widetilde{\boldsymbol{v}}$ and a displacement field of the above form $\widetilde{\boldsymbol{v}}=\boldsymbol{c}+\boldsymbol{d} \wedge \boldsymbol{\Theta}$ is called an infinitesimal rigid displacement of the set $\Theta(\Omega)$.

The infinitesimal rigid displacement lemma plays a crucial rôle for establishing the uniqueness (possibly in a quotient space) and, in conjunction with Korn's inequality, the existence of solutions to the boundary value problems of three-dimensional linearized elasticity expressed in terms of curvilinear coordinates; see [3, Chapter 1]. Note in passing that the more customary version, in Cartesian coordinates, of this lemma is immediately recovered by identifying $\mathbb{E}^{3}$ with $\mathbb{R}^{3}$ and by letting $\boldsymbol{\Theta}=\boldsymbol{i} \boldsymbol{d}_{\Omega}$.

One objective of this paper is to put this lemma in its proper perspective, as the linearized counterpart of the familiar rigidity theorem of threedimensional differential geometry, once this theorem has been properly extended to the Sobolev space $\boldsymbol{H}^{1}(\Omega)$.

This extension is carried out in Theorem 1, which relies in particular on a crucial extension of Liouville's theorem, originally due to Reshetnyak [8] and recently given a particularly concise and elegant proof by Friesecke, James and Müller [7]. 
It is then shown in Theorem 2 and its corollary that the set $\mathcal{M}_{\text {rig }}$ formed by all the rigid displacements of the set $\Theta(\Omega)$, i.e., those that satisfy the assumptions of the extended rigidity theorem, is a submanifold of dimension 6 and of class $C^{\infty}$ of the space $\boldsymbol{H}^{1}(\Omega)$.

It is finally established in Theorem 3 that the vector space spanned by the infinitesimal rigid displacements of the set $\boldsymbol{\Theta}(\Omega)$ is nothing but the tangent space at the origin to the manifold $\mathcal{M}_{\text {rig. }}$. This result hinges on the wellknown characterization of the tangent space at $\boldsymbol{I}$ to the special orthogonal group.

The results of this paper have been announced in [5]. Their extension to rigid and infinitesimal rigid displacements on a surface is carried out in [6].

\section{The rigidity theorem in Sobolev spaces}

All spaces, matrices, etc., considered are real. The notations $\mathbb{M}^{3}, \mathbb{O}^{3}, \mathbb{O}_{+}^{3}$, and $\mathbb{A}^{3}$ respectively designate the sets of all square matrices of order 3 , of all orthogonal matrices of order 3, of all matrices $\boldsymbol{Q} \in \mathbb{O}^{3}$ with $\operatorname{det} \boldsymbol{Q}=1$, and of all antisymmetric matrices of order 3 . The set $\mathbb{O}_{+}^{3}$ is the special orthogonal group. Given $\boldsymbol{A} \in \mathbb{M}^{3}$, Cof $\boldsymbol{A}$ designates the cofactor matrix of $\boldsymbol{A}$; thus $\operatorname{Cof} \boldsymbol{A}=(\operatorname{det} \boldsymbol{A}) \boldsymbol{A}^{-T}$ if $\boldsymbol{A}$ is invertible.

Latin indices range over the set $\{1,2,3\}$ and the summation convention with respect to repeated indices is used in conjunction with this rule. The identity mapping of a set $X$ is denoted $\boldsymbol{i} \boldsymbol{d}_{X}$.

The notation $\mathbb{E}^{3}$ designates a three-dimensional Euclidean space and $\boldsymbol{a} \cdot \boldsymbol{b}, \boldsymbol{a} \wedge \boldsymbol{b}$, and $|\boldsymbol{a}|=\sqrt{\boldsymbol{a} \cdot \boldsymbol{a}}$ respectively designate the Euclidean inner product, the exterior product of $\boldsymbol{a}, \boldsymbol{b} \in \mathbb{E}^{3}$, and the Euclidean norm of $a \in \mathbb{E}^{3}$.

Let $\Omega$ be an open subset of $\mathbb{R}^{3}$, let $x_{i}$ denote the coordinates of a point $x \in \mathbb{R}^{3}$, and let $\partial_{i}:=\partial / \partial x_{i}$ and $\partial_{i j}:=\partial^{2} / \partial x_{i} \partial x_{j}$. Let $\Theta \in \mathcal{C}^{1}\left(\Omega ; \mathbb{E}^{3}\right)$ be an immersion, i.e., a mapping such that the three vectors

$$
\boldsymbol{g}_{i}(x):=\partial_{i} \boldsymbol{\Theta}(x)
$$

are linearly independent at all points $x \in \Omega$. The metric tensor field $\left(g_{i j}\right) \in$ $\mathcal{C}^{0}\left(\Omega ; \mathbb{M}^{3}\right)$ of the set $\boldsymbol{\Theta}(\Omega)$ (which is open in $\mathbb{E}^{3}$ since $\boldsymbol{\Theta}$ is an immersion; see, e.g., [10, Theorem 3.8.10]) is defined by means of its covariant components

$$
g_{i j}(x):=\boldsymbol{g}_{i}(x) \cdot \boldsymbol{g}_{j}(x), \quad x \in \Omega,
$$

which are used in particular for computing lengths of curves inside the set $\Theta(\Omega)$, considered as isometrically imbedded in $\mathbb{E}^{3}$. Note that the symmetric 
positive definite matrix $\left(g_{i j}(x)\right) \in \mathbb{M}^{3}, x \in \Omega$, is also given by

$$
\left(g_{i j}(x)\right)=\nabla \Theta(x)^{T} \nabla \Theta(x),
$$

where $\boldsymbol{\nabla} \boldsymbol{\Theta}(x) \in \mathbb{M}^{3}$ is the matrix whose $j$-th column is $\boldsymbol{g}_{j}(x)$.

In order to avoid cumbersome statements, the distinction will not be made between an element in a Sobolev space, which is an equivalence class, and one of its elements.

The classical rigidity theorem for an open set asserts that, if two immersions $\widetilde{\boldsymbol{\Theta}} \in \mathcal{C}^{1}(\Omega):=\mathcal{C}^{1}\left(\Omega ; \mathbb{E}^{3}\right)$ and $\Theta \in \mathcal{C}^{1}(\Omega)$ have the same metric tensor fields, i.e., if $\widetilde{g}_{i j}=g_{i j}$ in $\Omega$ (with self-explanatory notations) and $\Omega$ is connected, then there exist a vector $c \in \mathbb{E}^{3}$ and a matrix $\boldsymbol{Q} \in \mathbb{O}^{3}$ such that

$$
\widetilde{\boldsymbol{\Theta}}(x)=\boldsymbol{c}+\boldsymbol{Q \Theta}(x) \text { for all } x \in \Omega .
$$

For a proof, see, e.g., [4, Theorem 3].

We now show that a similar result holds under the weaker assumption that $\widetilde{\boldsymbol{\Theta}} \in \boldsymbol{H}^{1}(\Omega):=H^{1}\left(\Omega ; \mathbb{E}^{3}\right)$.

The way the result in part (i) of the next proof is derived is due to Friesecke, James \& Müller [7]; the result itself goes back to Reshetnyak [8].

Theorem 1 (rigidity theorem) Let $\Omega$ be a connected open subset of $\mathbb{R}^{3}$ and let $\Theta \in \mathcal{C}^{1}(\Omega)$ be a mapping that satisfies $\operatorname{det} \nabla \Theta>0$ in $\Omega$. Assume that there exists a vector field $\widetilde{\boldsymbol{\Theta}} \in \boldsymbol{H}^{1}(\Omega)$ that satisfies

$$
\operatorname{det} \nabla \widetilde{\Theta}>0 \text { a.e. in } \Omega \text { and } \widetilde{g}_{i j}=g_{i j} \text { a.e. in } \Omega \text {. }
$$

Then there exist a vector $\boldsymbol{c} \in \mathbb{E}^{3}$ and a matrix $\boldsymbol{Q} \in \mathbb{O}_{+}^{3}$ such that

$$
\widetilde{\boldsymbol{\Theta}}(x)=\boldsymbol{c}+\boldsymbol{Q} \boldsymbol{\Theta}(x) \text { for almost all } x \in \Omega .
$$

Proof. The Euclidean space $\mathbb{E}^{3}$ is identified with the space $\mathbb{R}^{3}$ throughout the proof.

(i) To begin with, we consider the special case where $\boldsymbol{\Theta}=\boldsymbol{i} \boldsymbol{d}_{\Omega}$. In other words, we are given a mapping $\widetilde{\boldsymbol{\Theta}} \in \boldsymbol{H}^{1}(\Omega)$ that satisfies $\nabla \widetilde{\boldsymbol{\Theta}}(x) \in \mathbb{O}_{+}^{3}$ for almost all $x \in \Omega$. Hence

$$
\operatorname{Cof} \nabla \widetilde{\boldsymbol{\Theta}}(x)=(\operatorname{det} \nabla \widetilde{\boldsymbol{\Theta}}(x)) \nabla \widetilde{\boldsymbol{\Theta}}(x)^{-T}=\nabla \widetilde{\boldsymbol{\Theta}}(x)^{-T} \text { for almost all } x \in \Omega \text {, }
$$

on the one hand. Since, on the other hand,

$$
\operatorname{div} \operatorname{Cof} \nabla \widetilde{\Theta}=\mathbf{0} \text { in }\left(\mathcal{D}^{\prime}(B)\right)^{3}
$$


in any open ball $B$ such that $\bar{B} \subset \Omega$ (to see this, combine the classical Piola identity in the space $\mathcal{C}^{2}(\bar{B})$ with the density of $\mathcal{C}^{2}(\bar{B})$ in $H^{1}(B)$ ), we conclude that

$$
\Delta \widetilde{\boldsymbol{\Theta}}=\operatorname{div} \operatorname{Cof} \nabla \widetilde{\boldsymbol{\Theta}}=\mathbf{0} \text { in }\left(\mathcal{D}^{\prime}(B)\right)^{3} .
$$

Hence $\widetilde{\boldsymbol{\Theta}}=\left(\widetilde{\Theta}_{j}\right) \in\left(\mathcal{C}^{\infty}(\Omega)\right)^{3}$. For such mappings, the identity

$$
\Delta\left(\partial_{i} \widetilde{\Theta}_{j} \partial_{i} \widetilde{\Theta}_{j}\right)=2 \partial_{i} \widetilde{\Theta}_{j} \partial_{i}\left(\Delta \widetilde{\Theta}_{j}\right)+2 \partial_{i k} \widetilde{\Theta}_{j} \partial_{i k} \widetilde{\Theta}_{j},
$$

together with the relations $\Delta \widetilde{\Theta}_{j}=0$ and $\partial_{i} \widetilde{\Theta}_{j} \partial_{i} \widetilde{\Theta}_{j}=3$ in $\Omega$, shows that $\partial_{i k} \widetilde{\Theta}_{j}=0$ in $\Omega$. The assumed connectedness of $\Omega$ then implies that there exist a vector $c \in \mathbb{E}^{3}$ and a matrix $\boldsymbol{Q} \in \mathbb{O}_{+}^{3}$ (by assumption, $\nabla \widetilde{\boldsymbol{\Theta}}(x) \in \mathbb{O}_{+}^{3}$ for almost all $x \in \Omega$ ) such that

$$
\widetilde{\boldsymbol{\Theta}}(x)=\boldsymbol{c}+\boldsymbol{Q} \boldsymbol{o} \boldsymbol{x} \text { for almost all } x \in \Omega .
$$

(ii) We consider next the general case. Let $x_{0} \in \Omega$ be given. Since $\boldsymbol{\Theta}$ is an immersion, the local inversion theorem can be applied; there thus exist bounded open neighborhoods $U$ of $x_{0}$ and $\widehat{U}$ of $\boldsymbol{\Theta}\left(x_{0}\right)$ satisfying $\bar{U} \subset \Omega$ and $\{\widehat{U}\}^{-} \subset \boldsymbol{\Theta}(\Omega)$, such that the restriction $\boldsymbol{\Theta}_{U}$ of $\boldsymbol{\Theta}$ to $U$ can be extended to a $\mathcal{C}^{1}$-diffeomorphism from $\bar{U}$ onto $\{\widehat{U}\}^{-}$.

Let $\Theta^{-1}: \widehat{U} \rightarrow U$ denote the inverse mapping of $\boldsymbol{\Theta}_{U}$, which therefore satisfies $\widehat{\nabla} \Theta^{-1}(\widehat{x})=(\nabla \Theta(x))^{-1}$ for all $\widehat{x}=\boldsymbol{\Theta}(x) \in \widehat{U}$ (the notation $\widehat{\nabla}$ indicates that differentiation is carried out with respect to the variable $\widehat{x} \in$ $\widehat{U})$. Define the composite mapping

$$
\widehat{\boldsymbol{\Phi}}:=\widetilde{\boldsymbol{\Theta}} \cdot \Theta^{-1}: \widehat{U} \rightarrow \mathbb{R}^{3} .
$$

Since $\widetilde{\boldsymbol{\Theta}} \in \boldsymbol{H}^{1}(U)$ and $\boldsymbol{\Theta}^{-1}$ can be extended to a $\mathcal{C}^{1}$-diffeomorphism from $\{\widehat{U}\}^{-}$onto $\bar{U}$, it follows that $\widehat{\boldsymbol{\Phi}} \in \boldsymbol{H}^{1}(\widehat{U})$ and that

$$
\widehat{\nabla} \widehat{\mathbf{\Phi}}(\widehat{x})=\nabla \widetilde{\boldsymbol{\Theta}}(x) \widehat{\nabla} \boldsymbol{\Theta}^{-1}(x)=\nabla \widetilde{\boldsymbol{\Theta}}(x) \nabla \boldsymbol{\Theta}(x)^{-1}
$$

for almost all $\widehat{x}=\boldsymbol{\Theta}(x) \in \widehat{U}$ (see, e.g., [2, Chapter 3]). Hence the assumptions $\operatorname{det} \nabla \Theta>0$ in $\Omega$, det $\nabla \widetilde{\Theta}>0$ a.e. in $\Omega$, and $\widetilde{g}_{i j}=g_{i j}$ a.e. in $\Omega$, together imply that $\widehat{\nabla} \widehat{\boldsymbol{\Phi}}(\widehat{x}) \in \mathbb{O}_{+}^{3}$ for almost all $\widehat{x} \in \widehat{U}$. By (i), there thus exist $\boldsymbol{c} \in \mathbb{R}^{3}$ and $\boldsymbol{Q} \in \mathbb{O}_{+}^{3}$ such that

$$
\widehat{\mathbf{\Phi}}(\widehat{x})=\widetilde{\boldsymbol{\Theta}}(x)=\boldsymbol{c}+\boldsymbol{Q} \boldsymbol{o} \widehat{\boldsymbol{x}} \text { for almost all } \widehat{x}=\boldsymbol{\Theta}(x) \in \widehat{U},
$$

or equivalently, such that

$$
\boldsymbol{\Xi}(x):=\boldsymbol{\nabla} \widetilde{\boldsymbol{\Theta}}(x) \boldsymbol{\nabla} \boldsymbol{\Theta}(x)^{-1}=\boldsymbol{Q} \text { for almost all } x \in U .
$$


Since the point $x_{0} \in \Omega$ is arbitrary, this relation shows that $\boldsymbol{\Xi} \in \boldsymbol{L}_{\text {loc }}^{1}(\Omega)$. By a classical result from distribution theory (cf. [9, Section 2.6]), we conclude from the assumed connectedness of $\Omega$ that $\boldsymbol{\Xi}(x)=\boldsymbol{Q}$ for almost all $x \in \Omega$, and consequently that

$$
\widetilde{\boldsymbol{\Theta}}(x)=\boldsymbol{c}+\boldsymbol{Q \Theta}(x) \text { for almost all } x \in \Omega .
$$

Remarks. (1) The existence of $\widetilde{\boldsymbol{\Theta}} \in \boldsymbol{H}^{1}(\Omega)$ satisfying the assumptions of Theorem 1 thus implies that $\boldsymbol{\Theta} \in \boldsymbol{H}^{1}(\Omega)$ and $\widetilde{\boldsymbol{\Theta}} \in \mathcal{C}^{1}(\Omega)$.

(2) If $\widetilde{\boldsymbol{\Theta}} \in \mathcal{C}^{1}(\Omega)$, the assumptions $\operatorname{det} \boldsymbol{\nabla} \boldsymbol{\Theta}>0$ in $\Omega$ and $\operatorname{det} \nabla \widetilde{\boldsymbol{\Theta}}>0$ in $\Omega$ are no longer necessary; but then it can only be concluded that $\boldsymbol{Q} \in \mathbb{O}^{3}$ : This is the classical rigidity theorem, of which Liouville's theorem is the special case corresponding to $\boldsymbol{\Theta}=\boldsymbol{i} \boldsymbol{d}_{\Omega}$.

(3) By contrast, if the mapping $\widetilde{\boldsymbol{\Theta}}$ is assumed to be instead in the space $\boldsymbol{H}^{1}(\Omega)$ (as in the statement of Theorem 1), an assumption about the sign of $\operatorname{det} \nabla \widetilde{\Theta}$ becomes necessary. To see this, let (for instance) $\Omega$ be an open ball centered at the origin in $\mathbb{R}^{3}$ and let $\widetilde{\boldsymbol{\Theta}}(x)=x$ if $x_{1} \geq 0$ and $\widetilde{\boldsymbol{\Theta}}(x)=$ $\left(-x_{1}, x_{2}, x_{3}\right)$ if $x_{1} \leq 0$. Then $\widetilde{\boldsymbol{\Theta}} \in \boldsymbol{H}^{1}(\Omega)$ and $\boldsymbol{\nabla} \widetilde{\boldsymbol{\Theta}} \in \mathbb{O}^{3}$ a.e. in $\Omega$; yet there does not exist any orthogonal matrix such that $\widetilde{\boldsymbol{\Theta}}(x)=\boldsymbol{Q} \boldsymbol{o x}$ for all $x \in \Omega$, since $\widetilde{\boldsymbol{\Theta}}(\Omega) \subset\left\{x \in \mathbb{R}^{3} ; x_{1} \geq 0\right\}$.

(4) If a mapping $\Theta \in \mathcal{C}^{1}(\Omega)$ satisfies $\operatorname{det} \nabla \Theta>0$ in $\Omega$, then $\Theta$ is an immersion. Conversely, if $\Omega$ is a connected open set and $\Theta \in \mathcal{C}^{1}(\Omega)$ is an immersion, then either $\operatorname{det} \nabla \Theta>0$ in $\Omega$ or $\operatorname{det} \nabla \Theta<0$ in $\Omega$. The assumption that $\operatorname{det} \boldsymbol{\nabla} \Theta>0$ in $\Omega$ made in Theorem 1 is simply intended to fix ideas (a similar result clearly holds under the other assumption).

(5) A little further ado shows that the conclusion of Theorem 1 is still valid if $\widetilde{\boldsymbol{\Theta}} \in \boldsymbol{H}^{1}(\Omega)$ is replaced by the weaker assumption $\widetilde{\boldsymbol{\Theta}} \in \boldsymbol{H}_{\text {loc }}^{1}(\Omega)$.

\section{The submanifold of rigid displacements}

All the results needed below about submanifolds in infinite-dimensional Banach spaces are found in [1]. If $\mathcal{M}$ is a submanifold, the tangent space to $\mathcal{M}$ at $m \in \mathcal{M}$ is denoted $T_{m} \mathcal{M}$.

We now establish that the set $\mathcal{M}$ formed by all the mappings $\widetilde{\boldsymbol{\Theta}} \in$ $\boldsymbol{H}^{1}(\Omega)$ that satisfy the assumptions of the rigidity theorem for an open set (Theorem 1) is a finite-dimensional submanifold of the space $\boldsymbol{H}^{1}(\Omega)$. Note that the assumption $\boldsymbol{\Theta} \in \boldsymbol{H}^{1}(\Omega)$ has been added to those of Theorem 1, simply to guarantee that the set $\mathcal{M}$ is non-empty. 
We also characterize the tangent space to $\mathcal{M}$ at $\boldsymbol{\Theta}$. Another equally important characterization of the same tangent space, involving this time the linearized change of metric tensor, will be given in Theorem 3 .

The notations used here are the same as in Section 1; in particular, $\boldsymbol{H}^{1}(\Omega)=H^{1}\left(\Omega ; \mathbb{E}^{3}\right), \mathcal{C}^{1}(\Omega)=\mathcal{C}^{1}\left(\Omega ; \mathbb{E}^{3}\right)$, and $\widetilde{g}_{i j}$ and $g_{i j}$ designate the covariant components of the metric tensor fields respectively associated with the mappings $\widetilde{\boldsymbol{\Theta}}$ and $\boldsymbol{\Theta}$.

Theorem 2 Let $\Omega$ be a connected open subset of $\mathbb{R}^{3}$ and let $\boldsymbol{\Theta} \in \mathcal{C}^{1}(\Omega) \cap$ $\boldsymbol{H}^{1}(\Omega)$ be a mapping that satisfies $\operatorname{det} \boldsymbol{\nabla} \Theta>0$ in $\Omega$. Then the set

$$
\mathcal{M}:=\left\{\widetilde{\boldsymbol{\Theta}} \in \boldsymbol{H}^{1}(\Omega) ; \operatorname{det} \nabla \widetilde{\boldsymbol{\Theta}}>0 \text { and } \widetilde{g}_{i j}=g_{i j} \text { a.e. in } \Omega\right\}
$$

is a submanifold of class $\mathcal{C}^{\infty}$ and of dimension 6 of the space $\boldsymbol{H}^{1}(\Omega)$ and its tangent space at $\boldsymbol{\Theta}$ is given by

$$
T_{\Theta} \mathcal{M}=\left\{\widetilde{\boldsymbol{v}} \in \boldsymbol{H}^{1}(\Omega) ; \exists \boldsymbol{c} \in \mathbb{E}^{3}, \exists \boldsymbol{A} \in \mathbb{A}^{3}, \widetilde{\boldsymbol{v}}=\boldsymbol{c}+\boldsymbol{A} \Theta \text { a.e. in } \Omega\right\} .
$$

Proof. The linear mapping $\boldsymbol{f}$ defined by

$$
\boldsymbol{f}:(\boldsymbol{c}, \boldsymbol{F}) \in \mathbb{E}^{3} \times \mathbb{M}^{3} \rightarrow \boldsymbol{f}(\boldsymbol{c}, \boldsymbol{F}):=\boldsymbol{c}+\boldsymbol{F} \boldsymbol{\Theta} \in \boldsymbol{H}^{1}(\Omega)
$$

is injective. To see this, let $\boldsymbol{c} \in \mathbb{E}^{3}$ and $\boldsymbol{F} \in \mathbb{M}^{3}$ be such that $\boldsymbol{\chi}(x):=$ $\boldsymbol{c}+\boldsymbol{F} \boldsymbol{\Theta}(x)=\mathbf{0}$ for almost all $x \in \Omega$. Then $\boldsymbol{\nabla} \boldsymbol{\chi}(x)=\boldsymbol{F} \boldsymbol{\nabla} \boldsymbol{\Theta}(x)=0$ for almost all $x \in \Omega$ and thus $\boldsymbol{F}=\mathbf{0}$ since $\operatorname{det} \boldsymbol{\nabla} \Theta>0$ in $\Omega$ by assumption.

Consequently, the image $\boldsymbol{f}\left(\mathbb{E}^{3} \times \mathbb{M}^{3}\right)$ is a linear subspace of dimension 12 of $\boldsymbol{H}^{1}(\Omega)$ and $\boldsymbol{f}$ is a $\mathcal{C}^{\infty}$-diffeomorphism between $\mathbb{E}^{3} \times \mathbb{M}^{3}$ and $\boldsymbol{f}\left(\mathbb{E}^{3} \times \mathbb{M}^{3}\right)$, since $\boldsymbol{f}$ is linear and this image is finite-dimensional.

By the rigidity theorem (Theorem 1 ), the set $\mathcal{M}$ may be equivalently defined as

$$
\mathcal{M}=\boldsymbol{f}\left(\mathbb{E}^{3} \times \mathbb{O}_{+}^{3}\right) .
$$

Since $\mathbb{E} \times \mathbb{O}_{+}^{3}$ is a submanifold of class $\mathcal{C}^{\infty}$ and of dimension 6 of $\mathbb{E}^{3} \times \mathbb{M}^{3}$ (the special orthogonal group $\mathbb{O}_{+}^{3}$ is a submanifold of class $\mathcal{C}^{\infty}$ and of dimension 3 of $\mathbb{M}^{3}$ ) and submanifolds of class $\mathcal{C}^{\infty}$ are preserved by $\mathcal{C}^{\infty}$-diffeomorphisms, $\mathcal{M}$ is thus a submanifold of class $\mathcal{C}^{\infty}$ and of dimension 6 of $\boldsymbol{f}\left(\mathbb{E}^{3} \times \mathbb{M}^{3}\right)$.

Noting that the closed subspace $\boldsymbol{f}\left(\mathbb{E}^{3} \times \mathbb{M}^{3}\right)$ is "split" in $\boldsymbol{H}^{1}(\Omega)$ since any closed subspace of the Hilbert space $\boldsymbol{H}^{1}(\Omega)$ has a closed complement in it, we thus conclude that $\mathcal{M}$ is also a submanifold of class $\mathcal{C}^{\infty}$ and of dimension 6 of $\boldsymbol{H}^{1}(\Omega)$ (this conclusion immediately follows from the definition of a submanifold; see [1, Definition 3.2.1]). 
Since $\boldsymbol{f}$ is linear and $T_{\boldsymbol{I}} \mathbb{O}_{+}^{3}=\mathbb{A}^{3}$, the tangent space to $\mathcal{M}$ at $\boldsymbol{\Theta}$ is given by

$$
\begin{aligned}
T_{\boldsymbol{\Theta}} \mathcal{M} & =T_{\boldsymbol{f}(\mathbf{0}, \boldsymbol{I})} \boldsymbol{f}\left(\mathbb{E}^{3} \times \mathbb{O}_{+}^{3}\right)=\boldsymbol{f}\left(T_{(\mathbf{0}, \boldsymbol{I})}\left(\mathbb{E}^{3} \times \mathbb{O}_{+}^{3}\right)\right)=\boldsymbol{f}\left(\mathbb{E}^{3} \times \mathbb{A}^{3}\right) \\
& =\left\{\widetilde{\boldsymbol{v}} \in \boldsymbol{H}^{1}(\Omega) ; \exists \boldsymbol{c} \in \mathbb{E}^{3}, \exists \boldsymbol{A} \in \mathbb{A}^{3}, \widetilde{\boldsymbol{v}}=\boldsymbol{c}+\boldsymbol{A} \Theta \text { a.e. in } \Omega\right\},
\end{aligned}
$$

and the proof is complete.

Remark. Let $\Omega$ be a connected open subset of $\mathbb{R}^{3}$, let $\Theta \in \mathcal{C}^{1}(\Omega)$ be a mapping that satisfies $\operatorname{det} \nabla \Theta>0$ in $\Omega$, and let

$$
\mathcal{M}^{\prime}:=\left\{\widetilde{\boldsymbol{\Theta}} \in \boldsymbol{H}_{\text {loc }}^{1}(\Omega) ; \operatorname{det} \nabla \widetilde{\boldsymbol{\Theta}}>0 \text { and } \widetilde{g}_{i j}=g_{i j} \text { a.e. } \Omega\right\} .
$$

Then it can likewise be established under this weaker assumption on $\Theta$ that $\mathcal{M}^{\prime}$ is a Fréchet submanifold (see, e.g., [1, Section 3.1]) of class $\mathcal{C}^{\infty}$ and of dimension 6 of the Fréchet space $\boldsymbol{H}_{\mathrm{loc}}^{1}(\Omega)$.

In three-dimensional elasticity in curvilinear coordinates, the set $\boldsymbol{\Theta}(\Omega)$ is viewed as the reference configuration of a three-dimensional elastic body (under the additional assumption that the immersion $\Theta$ is injective, but this assumption is irrelevant for our present purposes). Then, for each $\widetilde{\boldsymbol{\Theta}} \in$ $\boldsymbol{H}^{1}(\Omega)$, the set $\widetilde{\boldsymbol{\Theta}}(\Omega)$ is viewed as a deformed configuration and the field $\widetilde{\boldsymbol{v}} \in \boldsymbol{H}^{1}(\Omega)$ defined by

$$
\widetilde{\boldsymbol{\Theta}}=\boldsymbol{\Theta}+\widetilde{\boldsymbol{v}}
$$

is viewed as a displacement field of the reference configuration $\boldsymbol{\Theta}(\Omega)$. If in particular $\widetilde{\boldsymbol{\Theta}} \in \mathcal{M}$, the field $\widetilde{\boldsymbol{v}}$ defined in this fashion is called a rigid displacement, and the subset $\mathcal{M}_{\text {rig }}$ of $\boldsymbol{H}^{1}(\Omega)$ defined by

$$
\mathcal{M}=\boldsymbol{\Theta}+\mathcal{M}_{\text {rig }}
$$

is accordingly called the manifold of rigid displacements (of the reference configuration $\Theta(\Omega)$ ). We now recast Theorem 2 in terms of the manifold $\mathcal{M}_{\text {rig }}$.

Corollary to Theorem 2 Let $\Omega$ be a connected open subset of $\mathbb{R}^{3}$, and let $\boldsymbol{\Theta} \in \mathcal{C}^{1}(\Omega) \cap \boldsymbol{H}^{1}(\Omega)$ be a mapping that satisfies $\operatorname{det} \boldsymbol{\nabla} \boldsymbol{\Theta}>0$ in $\Omega$. Then the manifold of rigid displacements of the set $\Theta(\Omega)$, viz.,

$$
\mathcal{M}_{\text {rig }}:=\left\{\widetilde{\boldsymbol{v}} \in \boldsymbol{H}^{1}(\Omega) ; \operatorname{det}(\boldsymbol{\nabla} \Theta+\boldsymbol{\nabla} \widetilde{\boldsymbol{v}})>0 \text { and } \widetilde{g}_{i j}=g_{i j} \text { a.e. in } \Omega\right\},
$$


is a submanifold of class $\mathcal{C}^{\infty}$ and of dimension 6 of the space $\boldsymbol{H}^{1}(\Omega)$ and its tangent space at $\mathbf{0}$ is given by

$$
\begin{aligned}
T_{\mathbf{0}} \mathcal{M}_{\text {rig }} & =T_{\Theta} \mathcal{M} \\
& =\left\{\widetilde{\boldsymbol{v}} \in \boldsymbol{H}^{1}(\Omega) ; \exists \boldsymbol{c} \in \mathbb{E}^{3}, \exists \boldsymbol{A} \in \mathbb{A}^{3}, \widetilde{\boldsymbol{v}}=\boldsymbol{c}+\boldsymbol{A} \Theta \text { a.e. in } \Omega\right\} .
\end{aligned}
$$

\section{The infinitesimal rigid displacement lemma in curvilinear coordinates revisited}

The covariant components of the linearized change of metric tensor associated with a displacement field $\widetilde{\boldsymbol{v}}$ of the set $\boldsymbol{\Theta}(\Omega)$, viewed as above as a reference configuration, are defined by

$$
e_{i \| j}(\widetilde{\boldsymbol{v}}):=\frac{1}{2}\left[\widetilde{g}_{i j}-g_{i j}\right]^{\text {lin }},
$$

where $g_{i j}$ and $\widetilde{g}_{i j}$ are the covariant components of the metric tensors of the sets $\boldsymbol{\Theta}(\Omega)$ and $\widetilde{\boldsymbol{\Theta}}(\Omega)$ where $\widetilde{\boldsymbol{\Theta}}:=\boldsymbol{\Theta}+\widetilde{\boldsymbol{v}}$, and $[\cdots]^{\text {lin }}$ denotes the linear part with respect to $\widetilde{\boldsymbol{v}}$ in the expression $[\cdots]$. A formal computation immediately gives

$$
e_{i \| j}(\widetilde{\boldsymbol{v}})=\frac{1}{2}\left(\partial_{i} \widetilde{\boldsymbol{v}} \cdot \boldsymbol{g}_{j}+\partial_{j} \widetilde{\boldsymbol{v}} \cdot \boldsymbol{g}_{i}\right), \text { where } \boldsymbol{g}_{i}=\partial_{i} \boldsymbol{\Theta} .
$$

This expression thus shows that

$$
e_{i \| j}(\widetilde{\boldsymbol{v}}) \in L_{\mathrm{loc}}^{2}(\Omega) \text { if } \widetilde{\boldsymbol{v}} \in \boldsymbol{H}^{1}(\Omega) \text { and } \boldsymbol{\Theta} \in \mathcal{C}^{1}(\Omega) .
$$

Under this assumption on the mapping $\boldsymbol{\Theta}$, a displacement field $\widetilde{\boldsymbol{v}} \in \boldsymbol{H}^{1}(\Omega)$ that satisfies $e_{i \| j}(\widetilde{\boldsymbol{v}})=0$ a.e. in $\Omega$ is called an infinitesimal rigid displacement of the set $\Theta(\Omega)$. Accordingly, the infinitesimal rigid displacement lemma in curvilinear coordinates stated in the Introduction consists in identifying the vector space $\mathcal{V}_{\text {rig }}^{\text {lin }}$ formed by such displacements.

The next theorem shows that this lemma has also a remarkably simple interpretation in terms of the manifold $\mathcal{M}_{\text {rig }}$ of rigid displacements introduced at the end of Section 2.

Theorem 3 Let $\Omega$ be a connected open subset of $\mathbb{R}^{3}$ and let $\boldsymbol{\Theta} \in \mathcal{C}^{1}(\Omega) \cap$ $\boldsymbol{H}^{1}(\Omega)$ be a mapping that satisfies $\operatorname{det} \boldsymbol{\nabla} \boldsymbol{\Theta}>0$ in $\Omega$. Then the space of infinitesimal rigid displacements of the set $\Theta(\Omega)$, viz.,

$$
\mathcal{V}_{\text {rig }}^{\operatorname{lin}}:=\left\{\widetilde{\boldsymbol{v}} \in \boldsymbol{H}^{1}(\Omega) ;\left[\widetilde{g}_{i j}-g_{i j}\right]^{\text {lin }}=0 \text { a.e. in } \Omega\right\},
$$


is given by

$$
\mathcal{V}_{\text {rig }}^{\text {lin }}=T_{\mathbf{0}} \mathcal{M}_{\text {rig }},
$$

where the tangent space $T_{\mathbf{0}} \mathcal{M}_{\text {rig }}$ has been identified in the Corollary to Theorem 2.

Proof. Let the vectors $\boldsymbol{g}^{k}$ be defined by means of the relations $\boldsymbol{g}^{k} \cdot \boldsymbol{g}_{\ell}=\delta_{\ell}^{k}$ in $\Omega$ and let $\left[\boldsymbol{g}^{k}\right]_{i}$ denote the $i$-th Cartesian component of the vector $\boldsymbol{g}^{k}$ in the space $\mathbb{E}^{3}$. Let $\widehat{\Omega}:=\boldsymbol{\Theta}(\Omega), \widehat{x}=\left(\widehat{x}_{i}\right)=\boldsymbol{\Theta}(x)$ for all $x \in \Omega, \widehat{\partial}_{i}:=\partial / \partial \widehat{x}_{i}$, and $\widehat{\partial}_{i j}:=\partial^{2} / \partial \widehat{x}_{i} \partial \widehat{x}_{j}$.

Given any $x_{0} \in \Omega$, there exists by the local inversion theorem an open and connected neighborhood $U$ of $x_{0}$ such that $\bar{U}$ is a compact subset of $\Omega$ and $\Theta$ restricted to $U$ can be extended to a $\mathcal{C}^{1}$-diffeomorphism from $\bar{U}$ onto $\{\widehat{U}\}^{-}$, where $\widehat{U}:=\boldsymbol{\Theta}(U)$. Let $\widehat{\boldsymbol{\Theta}}: \widehat{U} \rightarrow U$ denote the inverse mapping defined in this fashion. If a function $v$ belongs to $H^{1}(U)$, the function $\widehat{v}=v \circ \widehat{\boldsymbol{\Theta}}$ belongs to $H^{1}(\widehat{U})$ since the restriction of $\boldsymbol{\Theta}$ to $U$ and the mapping $\widehat{\Theta}$ are both Lipschitz-continuous on their domains of definitions.

Given a vector field $\widetilde{\boldsymbol{v}} \in \boldsymbol{H}^{1}(U)$, let the vector field $\widehat{\boldsymbol{v}}=\left(\widehat{v}_{i}\right) \in \boldsymbol{H}^{1}(\widehat{U})$ be defined by $\widehat{\boldsymbol{v}}(\widehat{x})=\widetilde{\boldsymbol{v}}(x)$ for almost all $\widehat{x}=\boldsymbol{\Theta}(x) \in \widehat{U}$. Then a simple computation based on the chain rule shows that the relations

$$
\widehat{e}_{i j}(\widehat{\boldsymbol{v}})(\widehat{x}):=\frac{1}{2}\left(\widehat{\partial}_{j} \widehat{v}_{i}+\widehat{\partial}_{i} \widehat{v}_{j}\right)(\widehat{x})=\left(e_{k \| \ell}(\widetilde{\boldsymbol{v}})\left[\boldsymbol{g}^{k}\right]_{i}\left[\boldsymbol{g}^{\ell}\right]_{j}\right)(x)
$$

hold for almost all $\widehat{x}=\boldsymbol{\Theta}(x) \in \widehat{U}$.

Hence the relations $e_{i \| j}(\widetilde{\boldsymbol{v}})=0$ in $U$ imply that $\widehat{e}_{i j}(\widehat{\boldsymbol{v}})=0$ in $\widehat{U}$. Consequently, the identity

$$
\widehat{\partial}_{j k} \widehat{v}_{i}=\widehat{\partial}_{j} \widehat{e}_{i k}(\widehat{\boldsymbol{v}})+\widehat{\partial}_{k} \widehat{e}_{i j}(\widehat{\boldsymbol{v}})-\widehat{\partial}_{i} \widehat{e}_{j k}(\widehat{\boldsymbol{v}}) \text { in } \mathcal{D}^{\prime}(\widehat{U})
$$

shows that $\widehat{\partial}_{j k} \widehat{v}_{i}=0$ in $\mathcal{D}^{\prime}(\widehat{U})$. By the same result in distribution theory as that used in the proof of Theorem 1, each function $\widehat{v}_{i}$ is thus a polynomial of degree $\leq 1$ (the set $\widehat{U}$ is connected). In other words, there exist a vector $\boldsymbol{c} \in \mathbb{E}^{3}$ and a matrix $\boldsymbol{A} \in \mathbb{M}^{3}$ such that $\widehat{\boldsymbol{v}}(\widehat{x})=\boldsymbol{c}+\boldsymbol{A} \boldsymbol{o} \widehat{\boldsymbol{x}}$ for almost all $\widehat{x} \in \widehat{U}$ and the relations $\widehat{e}_{i j}(\widehat{\boldsymbol{v}})=0$ further imply that $\boldsymbol{A} \in \mathbb{A}^{3}$. We thus conclude that

$$
\widetilde{\boldsymbol{v}}(x)=\boldsymbol{c}+\boldsymbol{A} \Theta(x) \text { for almost all } x \in U .
$$

Since the point $x_{0}$ is arbitrary and $\Omega$ is connected, this relation holds in fact for almost all $x \in \Omega$ (given any $x_{1} \in \Omega$, cover any path joining $x_{0}$ to $x_{1}$ 
by a finite number of neighborhoods similar to $U$ and repeat the previous argument). In other words,

$$
\mathcal{V}_{\text {rig }}^{\text {lin }}=\left\{\widetilde{\boldsymbol{v}} \in \boldsymbol{H}^{1}(\Omega) ; \exists \boldsymbol{c} \in \mathbb{E}^{3}, \exists \boldsymbol{A} \in \mathbb{A}^{3}, \widetilde{\boldsymbol{v}}=\boldsymbol{c}+\boldsymbol{A \Theta} \text { a.e. in } \Omega\right\} .
$$

The conclusion then follows from the Corollary to Theorem 2.

Remarks. (1) Theorem 3 indicates why an "infinitesimal" rigid displacement should preferably be called a "linearized" rigid displacement.

(2) The proof of Theorem 3 also shows that, under the weaker assumption $\boldsymbol{\Theta} \in \mathcal{C}^{1}(\Omega)$, a vector field $\widetilde{\boldsymbol{v}} \in \boldsymbol{H}_{\text {loc }}^{1}(\Omega)$ satisfies $e_{i \| j}(\widetilde{\boldsymbol{v}})=0$ a.e. in $\Omega$ if and only if there exist a vector $\boldsymbol{c} \in \mathbb{E}^{3}$ and a matrix $\boldsymbol{A} \in \mathbb{A}^{3}$ such that $\widetilde{\boldsymbol{v}}=\boldsymbol{c}+\boldsymbol{A \Theta}$ a.e. in $\Omega$.

The manifold of rigid displacements can be equivalently written as

$$
\mathcal{M}_{\text {rig }}=\left\{\widetilde{\boldsymbol{v}} \in \boldsymbol{H}^{1}(\Omega) ; \operatorname{det}(\boldsymbol{\nabla} \boldsymbol{\Theta}+\boldsymbol{\nabla} \widetilde{\boldsymbol{v}})>0 \text { a.e. in } \Omega, \mathcal{F}_{i j}(\widetilde{\boldsymbol{v}})=\mathbf{0} \text { a.e. in } \Omega\right\},
$$

where the mappings $\mathcal{F}_{i j}: \boldsymbol{H}^{1}(\Omega) \rightarrow L^{1}(\Omega)$ are defined by

$$
\mathcal{F}_{i j}(\widetilde{\boldsymbol{v}}):=\partial_{i}(\boldsymbol{\Theta}+\widetilde{\boldsymbol{v}}) \cdot \partial_{j}(\boldsymbol{\Theta}+\widetilde{\boldsymbol{v}})-\partial_{i} \boldsymbol{\Theta} \cdot \partial_{j} \boldsymbol{\Theta}, \quad \widetilde{\boldsymbol{v}} \in \boldsymbol{H}^{1}(\Omega) .
$$

Such mappings are clearly Fréchet-differentiable and their Gâteaux derivatives at $\mathbf{0}$ are given by $D \mathcal{F}_{i j}(\mathbf{0}) \widetilde{\boldsymbol{v}}=2 e_{i \| j}(\widetilde{\boldsymbol{v}})$ for all $\widetilde{\boldsymbol{v}} \in \boldsymbol{H}^{1}(\Omega)$, by definition of the functions $e_{i \| j}(\widetilde{\boldsymbol{v}})$. Theorem 3 thus shows that

$$
T_{\mathbf{0}} \mathcal{M}_{\text {rig }}=\left\{\widetilde{\boldsymbol{v}} \in \boldsymbol{H}^{1}(\Omega) ; D \mathcal{F}_{i j}(\mathbf{0}) \widetilde{\boldsymbol{v}}=0 \text { a.e. in } \Omega\right\} .
$$

In other words, the space $T_{\mathbf{0}} \mathcal{M}_{\text {rig }}$ has the expression that is naturally expected, albeit often delicate to establish, of the tangent space to a submanifold of an infinite-dimensional Banach space defined by means of equations; see in this respect [1, Chapter 3].

\section{Acknowledgment}

The work of the second author was partially supported by Research Project No. 9380037 from City University of Hong Kong, whose contribution is gratefully acknowledged.

\section{References}

[1] Abraham R., Marsden, J.E. and Ratiu, T.: Manifolds, Tensor Analysis, and Applications, Second Edition, Springer-Verlag, New York, 1988. 
[2] Adams, R.A.: Sobolev Spaces, Academic Press, New York, 1975.

[3] Ciarlet, P.G.: Mathematical Elasticity, Volume III: Theory of Shells, North-Holland, Amsterdam, 2000.

[4] Ciarlet, P.G. and Larsonneur, F.: On the recovery of a surface with prescribed first and second fundamental forms, J. Math. Pures Appl. 81 (2002), 167-185.

[5] Ciarlet, P.G. and Mardare, C.: On rigid displacements and their relation to the infinitesimal rigid displacement lemma in three-dimensional elasticity, C.R. Acad. Sci. Paris, Sér. I (to appear).

[6] Ciarlet, P.G. and Mardare, C.: On rigid and infinitesimal rigid displacements in shell theory (to appear).

[7] Friesecke, G., James, R.D. and Müller, S.: A theorem on geometric rigidity and the derivation of nonlinear plate theory from three dimensional elasticity, Comm. Pure Appl. Math. 55 (2002), 1461-1506.

[8] Reshetnyak, Y.G.: Liouville's theory on conformal mappings under minimal regularity assumptions, Sibirskii Math. J. 8 (1967), 69-85.

[9] Schwartz, L.: Théorie des Distributions, Hermann, Paris, 1966

[10] Schwartz, L.: Analyse II: Calcul Différentiel et Equations Différentielles, Hermann, Paris, 1992. 